\title{
FINITISTIC DIMENSION AND A HOMOLOGICAL GENERALIZATION OF SEMI-PRIMARY RINGS
}

BY

HYMAN BASS

Introduction. If $R$ is a ring and $M$ a left $R$-module, then homological algebra attaches three dimensions to $M$, projective, weak, and injective $\left.{ }^{1}\right)$. By taking the supremum of one of these dimensions as $M$ ranges over all left $R$-modules, one obtains one of the left "global" dimensions of $R$. Auslander and Buchsbaum [3] and, subsequently, Serre [14], found it relevant and fruitful, in the study of commutative Noetherian rings, to introduce a "finitistic global" dimension defined by restricting the supremum of projective dimensions to finitely generated modules of finite projective dimension. The impressive theory developed by these authors prompted Kaplansky to consider, for general commutative rings, a similar finitistic dimension (waiving the restriction, in the above, to finitely generated modules), and, in a seminar at the University of Chicago (1958), he proved the theorem below, characterizing commutative rings $R$ for which this dimension vanishes. This result is the origin of the present paper.

Definition. If $N$ is an ideal in a ring $R$, we say that $N$ is left $T$-nilpotent (" $T$ " for transfinite) if, given any sequence $\left\{a_{i}\right\}$ of elements in $N$, there exists an $n$ such that $a_{1} \cdots a_{n}=0$. (Right $T$-nilpotence requires instead that $a_{n} \cdots a_{1}=0$.)

THEOREM (KAPLANSKY). The following are equivalent for a commutative ring $R$.

(1) Every $R$-module has projective dimension 0 or $\infty$.

(2) $R$ is a direct sum of a finite number of local $\left({ }^{2}\right)$ rings, each with T-nilpotent maximal ideal.

Now, the (finitistic) global dimensions described above are obviously special examples of a whole family of (finitistic) global dimensions; we describe each by specifying the type of dimension and the modules allowed to contend in taking the supremum.

Part II of this paper presents the results of a general investigation of these dimensions. The principal results here are characterizations of rings for which various of these dimensions vanish, and, in some cases, equal one. In particular, we prove the noncommutative extension of Kaplansky's theorem

Received by the editors July 20, 1959.

(1) All rings have units, and all modules are unitary. For the notions of homological algebra, we refer to the ubiquitous Cartan-Eilenberg [5].

(2) By a "local" ring we mean only one with a unique maximal left ideal. 
(Theorem 6.3). These results are of the same genre as the theorems describing the vanishing of the ordinary global dimension [5, I, Theorem 4.2] and of the weak global dimension (Auslander [2], and Harada [9]). Corollary 7.3 puts into better perspective the observation (see, e.g., [1]) that the global dimension of a quasi-Frobenius ring is either 0 or $\infty$. Part II concludes in $\$ 8$ with the proofs of certain general inequalities relating the several global dimensions of a given ring, and it includes examples illustrating the preceding results.

There is another related, but perhaps more interesting result growing out of Kaplansky's theorem. This result provides one of those gratifying instances in which several ostensibly diverse notions are shown to be intimately related. Before stating the result, let us review the relevant background.

One easily sees that every module is an epimorphic image of a projective (even free) module. Dually, every module $M$ can be embedded in an injective module, though this is not as easily proved. In an elegant little paper [7], Eckmann and Schopf prove not only this, but that there is a minimal such embedding which is unique in a very strong sense. This minimal containing injective is called the injective envelope of $M$.

If we dualize the notion of an injective envelope, we rediscover what are essentially the "minimal epimorphisms" of Eilenberg and Nakayama [9], and Eilenberg [8]. However, it is convenient for us to alter slightly the definition of minimal epimorphism here. We shall call the dual of an injective envelope a "projective cover." In contrast with the Eckmann Schopf theorem, projective covers seldom exist; e.g. an abelian group ( $Z$-module) has a projective cover only if it is free. Their usefulness is established in Eilenberg's homological dimension and syzygies [8] wherein he studies the dimension theory for modules having projective covers. Eilenberg calls a category of modules "perfect" if every module in it has a projective cover. Thus, we call a ring $R$ left perfect if every left $R$-module has a projective cover. As an interesting consequence of Eilenberg's results, if $R$ is left perfect, then every left $R$-module has the same weak as projective dimension.

We use the following terminology in the sequel: If $R$ is a ring, the Jacobson radical is denoted $J$-radical, and we call $R J$-semi-simple if the $J$-radical is zero. Semi-simple shall mean $J$-semi-simple and Artinian (i.e. with both minimum conditions). The socle of a module is the sum of all simple submodules.

Theorem P. Let $R$ be a ring, $N$ its $J$-radical. Then the following are equivalent.

(1) $N$ is left $T$-nilpotent and $R / N$ is semi-simple.

(2) $R$ is left perfect.

(3) Every left $R$-module has the same weak as projective dimension.

(4) A direct limit of left $R$-modules of projective dimension $\leqq n$ has projective dimension $\leqq n$. 
(5) A direct limit of projective left $R$-modules is projective.

(6) $R$ satisfies the descending chain condition on principal right ideals.

(7) $R$ has no infinite sets of orthogonal idempotents, and every nonzero right $R$-module has nonzero socle.

The basic idea for this theorem came from the observation of S. Schanuel that the rings described by Kaplansky's theorem are precisely the commutative perfect rings. Moreover, we note that after the author communicated the implications (1) $\Leftrightarrow(2)$ (more precisely, Theorem 2.1) to Dock Sang Rim, the latter developed proofs of them independently in a seminar at Columbia University.

Part I of this paper is devoted to the proof of Theorem P. Theorem 2.1 generalizes the implications $(1) \Leftrightarrow(2)$, and $\S 3$ provides various examples, including a ring which is left, but not right, perfect.

This paper is an expansion of portions of the author's dissertation at the University of Chicago, and it was written during his tenure as a National Science Foundation Fellow. The author is deeply indebted to Professor Kaplansky as the source of most of the ideas and several of the proofs in what follows. He is also grateful to S. Chase and S. Schanuel for numerous stimulating conversations.

\section{Part I. The Proof of Theorem P}

1. $(3) \Rightarrow(4) \Rightarrow(5) \Rightarrow(6) \Rightarrow(7) \Rightarrow(1)$. We prove Theorem $P$ by first establishing the above implications in this section. Then in $\$ 2$ we prove a theorem which includes the implications $(1) \Leftrightarrow(2)$. Finally, using information from these arguments, we prove $(2) \Rightarrow(3)$ at the end of $\S 2$.

Notation. If $A$ is a left (right) $R$-module, we shall denote by $P d_{R}(A)$, $W d_{R}(A)$, and $I d_{R}(A)$ the projective, weak, and injective dimensions, respectively, of $A$ as a left (right) $R$-module.

(3) implies (4). Let $A=\operatorname{Lim}_{\rightarrow} A_{\alpha}$ with $P d_{R}\left(A_{\alpha}\right) \leqq n$ for all $\alpha$. Then $W d_{R}\left(A_{\alpha}\right)$ $\leqq n$ for all $\alpha$, so, since Tor ${ }^{R}$ commutes with direct limits, $W d_{R}(A) \leqq n$. Therefore $P d_{R}(A) \leqq n$, by (3).

(4) implies (5) is trivial.

We base the proof that (5) implies (6) on a close analysis of a special type of projective resolution. This information, to which we shall refer several times, is recorded in the following three lemmas.

Notation. Let $\left\{a_{n}\right\}_{n=1,2, \ldots}$ be any sequence of elements in a ring $R$. Then we denote by $\left[F,\left\{a_{n}\right\}, G\right]$

(i) A free left $R$-module $F$ with basis $x_{1}, x_{2} \cdots$, and

(ii) The submodule $G$ of $F$ generated by $\left\{x_{n}-a_{n} x_{n+1}\right\}_{n=1,2}, \ldots$. The image of $x_{n}$ in $F / G$ we denote by $z_{n}$.

Lemma 1.1. $P d_{R}(F / G) \leqq 1$, and $F / G$ is a direct limit of projective modules, so $W d_{R}(F / G)=0$. 
Proof. Let $G_{n}$ be the submodule of $F$ generated by $x_{1}-a_{1} x_{2}, \cdots, x_{n}$ $-a_{n} x_{n+1}$. We see easily that these are free generators of $G_{n}$ and that $F / G_{n}$ is free. Therefore, $G$ has $\left\{x_{n}-a_{n} x_{n+1}\right\}$ as a free basis, so $P d_{R}(F / G) \leqq 1$. Moreover, $F / G=\operatorname{Lim}_{n \rightarrow \infty} F / G_{n}$.

Lemma 1.2. If $J_{k}=\left\{r \in R \mid r a_{k} \cdots a_{k+n}=0\right.$ for some $\left.n\right\}$, then $\left((0): z_{k}\right)=J_{k}$.

Proof. We see this by viewing $F / G$ as a direct limit, or with a coordinate argument, as follows. Clearly $J_{k} \subset\left((0): z_{k}\right)$ since $z_{k}=a_{k} z_{k+1}=\cdots=a_{k} \cdots$ $a_{k+n} z_{k+n+1}=\cdots$. Suppose $r z_{k}=0$; i.e. $r x_{k} \in G$. Then

$$
r x_{k}=\sum_{i} r_{i}\left(x_{i}-a_{i} x_{i+1}\right) \text {. }
$$

By comparing coefficients of the $x_{i}$ we see that $r_{i}=0$ for $i<k$ and

$$
\begin{aligned}
r & =r_{k} \\
0 & =r_{k+1}-r_{k} a_{k} \\
& \vdots \\
0 & =r_{k+n}-r_{k+n-1} a_{k+n-1} .
\end{aligned}
$$

Thus, $\quad r_{k+n}=r_{k+n-1} a_{k+n-1}=r_{k+n-2} a_{k+n-2} a_{k+n-1}=\cdots=r_{k} a_{k} \cdots a_{k+n-1}$ $=r a_{k} \cdots a_{k+n-1}$. But, for sufficiently large $n, r_{k+n}=0$.

Lemma 1.3. Suppose $G$ is a direct summand of $F$. Then the chain $\left\{a_{1} \cdots a_{n} R\right\}$ of principal right ideals terminates.

Proof. Identifying $F / G$ with a direct summand of $F$, we may write $F=F / G \oplus G$ and $x_{n}=z_{n}+g_{n}$, with $g_{n} \in G$. If we expand

$$
z_{n}=c_{n 1} x_{1}+\cdots+c_{n k} x_{k}+\cdots
$$

then the row finite matrix $\left(\left(c_{i j}\right)\right)$ is idempotent, since it defines the endomorphism which projects $F$ onto $F / G$. Let $I$ be the right ideal generated by $\left\{c_{11}, c_{12}, \cdots, c_{1 n}, \cdots\right\}$, the coordinates of $z_{1}$. Since $z_{1}=a_{1} z_{2}=\cdots=a_{1} \cdots$ $a_{n} z_{n+1}=\cdots$ we have $I \subset \bigcap_{n}\left(a_{1} \cdots a_{n} R\right)$. We shall prove the lemma by showing that, for some $m, a_{1} \cdots a_{m} \in I$.

Since $\left(\left(c_{i j}\right)\right)$ is idempotent, we have, for sufficiently large $n$, and for all $j$,

$$
c_{1 j}=\sum_{k=1}^{n+1} c_{1 k} c_{k j}
$$

From $z_{k}=a_{k} \cdots a_{n} z_{n+1}$ we have $c_{k j}=a_{k} \cdots a_{n} c_{n+1 j}$ for $k \leqq n$ and for all $j$. Therefore, for all $j$,

$$
\begin{aligned}
c_{1 j} & =\sum_{k=1}^{n+1} c_{1 k} a_{k} \cdots a_{n} c_{n+1 j} \\
& =\left(\sum_{k=1}^{n+1} c_{1 k} a_{k} \cdots a_{n}\right) c_{n+1 j} .
\end{aligned}
$$


Let $\gamma=\sum_{k=1}^{n+1} c_{1 k} a_{k} \cdots a_{n}$. Then these equations assert that $z_{1}=\gamma z_{n+1}$. Therefore, by (1.2), $\gamma-a_{1} \cdots a_{n} \in J_{n+1}$; i.e. for some $h \geqq 1,\left(\gamma-a_{1} \cdots a_{n}\right) a_{n+1} \cdots$ $a_{n+h}=0$. But then, if we set $m=n+h, a_{1} \cdots a_{m}=\gamma a_{n+1} \cdots a_{n+h} \in I$ since $\gamma \in I$.

Now we resume the proof of Theorem $\mathrm{P}$.

(5) implies (6). Any decreasing chain of principal right ideals in $R$ is clearly expressible in the form $\left\{a_{1} \cdots a_{n} R\right\}$ for some sequence $\left\{a_{n}\right\}$ in $R$. Form $\left[F,\left\{a_{n}\right\}, G\right]$ as above. By (1.1), $F / G$ is a direct limit of projectives, so by (5), $F / G$ is itself projective. Therefore, $G$ is a direct summand of $F$, so, by (1.3), the chain $\left\{a_{1} \cdots a_{n} R\right\}$ terminates.

(6) implies (7). Clearly any infinite set of orthogonal idempotents gives rise to an infinite decreasing chain of principal right ideals.

Now let $A$ be a right $R$-module and $0 \neq \alpha \in A$. If $\alpha R$ is not simple there exists an $a_{1} \in R$ such that $(0) \neq \alpha a_{1} R \neq \alpha R$. If $\alpha a_{1} R$ is not simple, there exists an $a_{2} \in R$ such that $(0) \neq \alpha a_{1} a_{2} R \neq \alpha a_{1} R$. If, continuing in this way, we never reach a simple module, we produce a chain

$$
\alpha R \neq \alpha a_{1} R \neq \cdots \neq \alpha a_{1} \cdots a_{n} R \neq \cdots .
$$

By (6) we know that, for some $n, a_{1} \cdots a_{n}=a_{1} \cdots a_{n+1} r$. Therefore, $\alpha a_{1} \cdots a_{n} \in \alpha a_{1} \cdots a_{n+1} R$; contradiction.

(7) implies (1). Let $N$ be the $J$-radical of $R$ and define inductively $N_{0}=(0), N_{\alpha+1}$ is such that $N_{\alpha+1} / N_{\alpha}$ is the socle of the right $R$-module $N / N_{\alpha}$, and, if $\alpha$ is a limit ordinal, $N_{\alpha}=\bigcup_{\beta<\alpha} N_{\beta}$. We know, by (7), that for some $\alpha_{0}, N=N_{\alpha_{0}}$. Therefore, if $a \in N$, we can define $h(a)$ to be the least $\alpha$ such that $a \in N_{\alpha}$. Note that $h(a)$ can never be a limit ordinal since, if $a \in \mathrm{U}_{\beta<\alpha} N_{\beta}$, then $a \in N_{\beta}$ for some $\beta<\alpha$. Therefore, if $a \neq 0$, we may write $h(a)=\beta+1$ for some $\beta$. Now, since $N_{\beta+1} N \subset N_{\beta}$, we have, for any $b \in N, h(a b)<h(a)$.

Suppose given a sequence $\left\{a_{n}\right\}$ of elements of $N$. Then if $a_{1} \cdots a_{n} \neq 0$ for all $n,\left\{h\left(a_{1} \cdots a_{n}\right)\right\}$ is an infinite strictly decreasing chain of ordinals, a familiar impossibility. Thus, $N$ is a left $T$-nilpotent. In particular, since $N$ is nil, it is well known that the nonexistence of an infinite orthogonal set of idempotents is inherited on $R / N$. We shall show that $R / N$ has minimum condition by showing that $R / N$ equals its right socle, $S$. In fact, since $R / N$ is $J$-semi-simple, each minimal right ideal is generated by an idempotent. It therefore follows from the above remarks that $S$ must be a finite direct sum of minimal right ideals, so $S$ is itself a direct summand of $R / N$. But then the complementary summand is a right $R$-module with zero socle, so is zero by (7).

REMARKs. (i) The requirement on idempotents cannot be dropped in condition (7), for the (weak) direct sum of infinitely many copies of a field (together with the identity) satisfies the weaker hypothesis.

(ii) Condition (7) has a natural dual which asserts, in addition to the condition on idempotents, that every nonzero left $R$-module has a simple epimorphic image. Left perfect rings have this property, and a ring with this 
property must have left $T$-nilpotent $J$-radical. Thus, to prove that the latter are left perfect one may reduce to the $J$-semi-simple case. We do not know whether this condition characterizes left perfect rings.

2. Perfect and semi-perfect rings: $(1) \Leftrightarrow(2) \Rightarrow(3)$. In this section we prove $(1) \Leftrightarrow(2)$, so we must first make precise the various concepts discussed.

Let $M$ be a module, $K$ a submodule. We call $K$ essential in $M$ if, for a submodule $S$ of $M, S \cap K=(0) \Rightarrow S=(0)$. We call $K$ superfluous in $M$ if, for a submodule $S$ of $M, S+K=M \Rightarrow S=M$. Given a homomorphism $f: A \rightarrow B$, we call $f$ essential if $\operatorname{im} f$ is essential in $B$; we call $f$ minimal if $\operatorname{ker} f$ is superfluous in $A$. An injective envelope of $M$ is an essential monomorphism of $M$ into an injective module. A projective cover of $M$ is a minimal epimorphism of a projective module onto $M$. We reserve the notation $E(M)$ and $P(M)$ for these respective objects. Thus, $E(M)$ is an injective module which we regard as containing $M$, and $M$ is essential in $E(M) . P(M)$ denotes a projective module together with an (undenoted) minimal epimorphism $P(M) \rightarrow M \rightarrow(0)$. We call a ring $R$ left perfect if every left $R$-module has a projective cover, and we call $R$ left semi-perfect if every cyclic left $R$-module has a projective cover.

The equivalence $(1) \Leftrightarrow(2)$ of Theorem $\mathrm{P}$ is the last statement of Theorem 2.1 below. With the information from the proof of Theorem 2.1 we easily conclude the proof of Theorem P at the end of this section by showing (2) $\Rightarrow(3)$.

TheOREM 2.1. Let $R$ be a ring, $N$ its $J$-radical. Then the following are equivalent.

(a) $R$ is left semi-perfect.

(a') $R$ is right semi-perfect.

(b) $R / N$ is semi-simple and idempotents can be lifted modulo $N$.

(c) Every finitely generated left $R$-module has a projective cover.

(c') Every finitely generated right $R$-module has a projective cover.

Moreover, $R$ is left perfect if and only if, in addition to (b), $N$ is left T-nilpotent.

Since (b) is left-right symmetric, and (c) trivially implies (a), it suffices that we prove $(a) \Rightarrow(b),(b) \Rightarrow(c)$, and, finally, the last statement of the theorem. We shall carry out the proof in this order, first preparing for $(a) \Rightarrow(b)$ with the following four lemmas.

Lemma 2.2. Let $I$ be a two sided ideal in $R$. Then if $P \rightarrow A \rightarrow(0)$ is an $R$ projective cover of an $R / I$-module $A$, the induced map $P / I P \rightarrow A \rightarrow(0)$ is an $R / I$-projective cover of $A$. Consequently, if $R$ is (semi) perfect, so also is $R / I$.

Proof. Let $K=\operatorname{ker}(P \rightarrow A)$. Since $I A=(0), I P \subset K$ and the second map is well defined. Moreover, $P / I P$ is $R / I$-projective. If $S / I P+K / I P=P / I P$ then $S+K=P$, so $S=P$ and therefore $S / I P=P / I P$; i.e. $P / I P \rightarrow A$ is minimal. 
Lemma 2.3. (Uniqueness of projective covers.) Suppose $(0) \rightarrow K \rightarrow P$ $\rightarrow A \rightarrow(0)$ is exact with $P$ projective and $P(A) \rightarrow A \rightarrow(0)$ is a projective cover. Then we can write $P=P(A) \oplus P^{\prime}$ with $P^{\prime} \subset K$ and $K \cap P(A)$ superfluous in $P(A)$.

Proof. Since $P$ is projective, there exists a map $P \rightarrow P(A)$ making

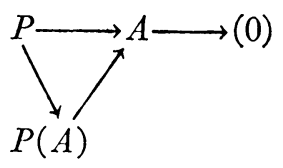

commutative. Since $\operatorname{im}(P \rightarrow P(A))+\operatorname{ker}(P(A) \rightarrow A)=P(A), \quad \operatorname{im}(P \rightarrow P(A))$ $=P(A)$, so $P \rightarrow P(A)$ is an epimorphism and therefore splits. (We have used the two defining properties of $P(A)$.) Thus, identifying $P(A)$ with a direct summand of $P$, we may write $P=P(A) \oplus P^{\prime}$, where $P^{\prime}=\operatorname{ker}(P \rightarrow P(A))$ $C \operatorname{ker}(P \rightarrow A)=K$. Moreover, $P \rightarrow A$ induces the given minimal epimorphism $P(A) \rightarrow A$ on $P(A)$, and the induced kernel is $K \cap P(A)$. From this the last statement follows.

Lemma 2.4. If $I$ is a left ideal of $R$, then $R \rightarrow{ }^{\text {nat }} R / I \rightarrow(0)$ is minimal if and only if $I \subset N$ (the $J$-radical). Moreover, if $R$ is left semi-perfect, either $I \subset N$ or $I$ contains a nonzero direct summand of $R$.

Proof. $I$ is superfluous in $R$ if and only if $I$ is comaximal with no proper left ideal, i.e. if and only if $I$ is contained in every maximal left ideal.

Suppose now that $R$ is left semi-perfect, so that $R / I$ has projective cover. Then, by (2.3), we can write $R=P(R / I) \oplus P^{\prime}$ with $P^{\prime} \subset I$ and $I \cap P(R / I)$ superfluous in $P(R / I)$. If $P^{\prime} \neq(0)$ we are finished. Otherwise $P(R / I)=R$, so $I \subset N$ by the first part of the lemma.

Lemma 2.5. If $R$ is $J$-semi-simple and left semi-perfect then $R$ has minimum condition.

Proof. We shall establish this by showing that $R$ equals its left socle, $S$. If not, $S \subset M$ for some maximal left ideal $M$. Applying (2.3) to the exact sequence $(0) \rightarrow M \rightarrow R \rightarrow R / M \rightarrow(0)$ we have $R=P \oplus Q$ with $Q \subset M$ and $M \cap P$ superfluous in $P$. The latter condition guarantees that $M \cap P$ can contain no direct summand of $P$, so also of $R$. Therefore, by the $J$-semi-simplicity and the last part of (2.4), $M \cap P=(0)$. But then $P \cong R / M$ so $P \subset S$; contradiction.

Now we are prepared to prove: (a) implies (b). If $R$ is semi-perfect so also is $R / N$, by (2.2). Therefore, by (2.5), $R / N$ has minimum condition. Now we see easily that the ability to lift idempotents modulo $N$ is tantamount to being able to lift direct sum decompositions of $R / N$ to decompositions of $R$. Suppose $R / N=A \oplus B$, and let $P \rightarrow A \rightarrow(0)$ and $Q \rightarrow B \rightarrow(0)$ be projective covers. Then we check easily that $P \oplus Q \rightarrow A \oplus B \rightarrow(0)$ is a projective cover. But, by (2.4), $R \rightarrow R / N=A \oplus B \rightarrow(0)$ is a projective cover. There- 
fore, $R=P \oplus Q$, by the uniqueness of the cover, and we have lifted the decomposition of $R / N$.

The proof that (b) implies (d) and of half the final statement of Theorem 2.1 is based on the following lemma which is essentially proved in Eilenberg's paper [8].

Lemma 2.6. Suppose $R / N$ is semi-simple and idempotents can be lifted modulo $N$. Then for a left $R$-module $A$ to have a projective cover, it suffices that for any left $R$-module $B$ requiring no more generators than $A, B=N B \Rightarrow B=(0)$.

Proof. Our hypotheses permit us to write $A / N A=\sum_{i} \oplus R e_{i} / N e_{i}$, where $e_{i}$ is an idempotent in $R$. Let $P=\sum_{i} \oplus R e_{i}$. Since $P$ is projective, there is a map $P \rightarrow A$ making

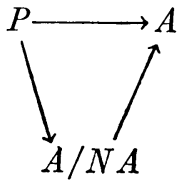

commutative. We claim $P \rightarrow A$ is a projective cover. If we show that $A \rightarrow A / N A$ is minimal (i.e. that $N A$ is superfluous in $A$ ) it will follow that $P \rightarrow A$ is an epimorphism. To show then that $P \rightarrow A$ is minimal, since, $\operatorname{ker}(P \rightarrow A) \subset \operatorname{ker}(P \rightarrow A / N A)=N P$, it will suffice to show $N P$ is superfluous in $P$. Thus we conclude the lemma by showing that if $C=$ either $A$ or $P$, and $S$ is a submodule such that $S+N C=C$, then $S=C$; i.e. $C / S=(0)$. But since $C / S$ clearly requires no more generators than $A$, this follows from the fact that $N(C / S)=C / S$.

Now we prove, $(\mathrm{b}) \Rightarrow(\mathrm{c})$. We need only observe that for any finitely generated left $R$-module $B, B=N B \Rightarrow B=(0)$. This is one form of "Nakayama's Lemma."

We use Lemma 2.6 also to prove: if $R / N$ is semi-simple and $N$ is left $T$-nilpotent, then $R$ is left perfect. Since idempotents can be lifted modulo any nil ideal, we need only show that for any module $B \neq(0), B \neq N B$.

Suppose $0 \neq \alpha \in B$ and $B=N B$. Then

$$
\alpha=\sum_{i_{1}} a_{i_{1}} \alpha_{i_{1}}, \quad a_{i_{1}} \in N, \quad \alpha_{i_{1}} \in B .
$$

Again, for each $i_{1}$

$$
\alpha_{i_{1}}=\sum_{i_{2}} a_{i_{1} i_{2}} \alpha_{i_{1} i_{2}}, \quad a_{i_{1} i_{2}} \in N, \quad \alpha_{i_{1} i_{2}} \in B .
$$

We continue indefinitely in this way;

$$
\alpha_{i_{1} \cdots i_{n-1}}=\sum_{i} a_{i_{1} \cdots i_{n}} \alpha_{i_{1} \cdots i_{n}}, \quad a_{i_{1} \cdots i_{n}} \in N, \quad \alpha_{i_{1} \cdots i_{n}} \in B .
$$

Then for each $n$, 


$$
\alpha=\sum_{i_{1}, i_{2}, \cdots, i_{n}} a_{i_{1}} a_{i_{1} i_{2}} \ldots a_{i_{1} \cdots i_{n}} \alpha_{i_{1}} \cdots i_{n},
$$

so, there is a sequence $i_{1 o}, \cdots, i_{n o}$ such that

$$
a_{i_{10}} a_{i_{10} i_{2 o}} \cdots a_{i_{10} i_{20} \cdots i_{n o}} \neq 0 .
$$

Letting such sequences be the vertices of a tree, in which an edge corresponds to adjoining one new index, we see that each vertex has finite index, and there exist paths of arbitrary length. Now by the Konig Graph Theorem we conclude that there exists an infinite path; i.e. there exists a sequence $i_{1 o}, i_{2 o}, \cdots, i_{n o} \cdots$ such that for all $n$,

$$
a_{i_{10}} a_{i_{10} i_{2 o}} \cdots a_{i_{10} \cdots i_{n o}} \neq 0 .
$$

This contradicts the $T$-nilpotence of $N$. QED.

To conclude the proof of Theorem 2.1, it remains only to show, conversely, that if $R$ is left perfect, then $R / N$ is semi-simple and $N$ is left $T$-nilpotent. The semi-simplicity is contained in the implication $(a) \Rightarrow(b)$ which we have already proved. For the latter we require the following useful result.

Proposition 2.7. Let $R$ be any ring, $N$ its $J$-radical, and $P$ a nonzero projective left $R$-module. Then $P \neq N P$.

Proof. Suppose $P \oplus Q=F$, a free module with a basis $\left\{x_{i}\right\}$ and $P \subset N F$. Writing $x_{i}=y_{i}+z_{i}, y_{i} \in P, z_{i} \in Q$, and expanding $y_{i}=\sum_{j} a_{i j} x_{j}$, we have $a_{i j} \in N$ for all $i$ and $j$. Since $z_{i}=x_{i}-y_{i}, z_{i}=\sum_{j}\left(\delta_{i j}-a_{i j}\right) x_{j}$. We shall show that the $z_{i}$ 's are linearly independent. For suppose $z_{1}, \cdots, z_{n}$ is any finite set of them. If we project $F$ onto the coordinates of $x_{1}, \cdots, x_{n}$, then the $z_{i}^{\prime}$ 's map onto elements $z_{i}^{\prime}$. We assert even that $z_{1}^{\prime}, \cdots, z_{n}^{\prime}$ are linearly independent. For

$$
z_{i}^{\prime}=\sum_{=1}^{n}\left(\delta_{i j}-a_{i j}\right) x_{j} ; \quad i=1, \cdots, n .
$$

Now, if $I$ is the $n \times n$ identity matrix, and $A=\left(\left(a_{i j}\right)\right)_{i, j=1, \cdots, n}$, then, by [10, I, $\S 7$, Theorem 3], $A$ belongs to the $J$-radical of the $n \times n$ matrix ring, so $I-A$ is invertible; in particular its rows are linearly independent. But the rows of $I-A$ are the coordinate vectors of the $z_{i}^{\prime}$.

Now suppose $a_{1} x_{1}+\cdots+a_{n} x_{n} \in P$. Then by projecting $F$ onto $Q$ (with kernel $P$ ), we see that $a_{1} z_{1}+\cdots+a_{n} z_{n}=0$. Therefore $a_{1}=\cdots=a_{n}=0$; i.e. $P=(0)$.

REMARK. One can show easily that for any projective module $P, N P$ is the intersection of the maximal submodules of $P$. Thus, an equivalent formulation of 2.7 asserts that "every nonzero projective module has a maximal submodule."

Now, we will show that if $R$ is left perfect, then $N$ is left $T$-nilpotent. Let $\left\{a_{n}\right\}$ be a sequence of elements in $N$ and form $\left[F,\left\{a_{n}\right\}, G\right]$ (see beginning 
of $\S 1$ ). Applying (2.3) to the exact sequence

$$
(0) \rightarrow G \rightarrow F \rightarrow F / G \rightarrow(0)
$$

we may write $F=P \oplus Q$ with $Q \subset G$ and $G \cap P$ superfluous in $P$. Clearly, $F=G+N F$, so

$$
\begin{aligned}
F & =G+N F \\
& =[(G \cap P) \oplus Q]+[N P \oplus N Q] \\
& =[(G \cap P)+N P] \oplus[Q+N Q] \\
& =[(G \cap P)+N P] \oplus Q .
\end{aligned}
$$

Therefore, $P=(G \cap P)+N P$. But, since $G \cap P$ is superfluous in $P, P=N P$. Finally, by (2.9), $P=(0)$; i.e. $G=F$. Hence, since $1 z_{1}=0, a_{1} \cdots a_{n}=0$ for some $n$, by (1.2). QED.

Theorem 2.1 is now proved.

We proceed finally to conclude the proof of Theorem $\mathrm{P}$. We must prove (2) implies (3); i.e. if $R$ is left perfect and $A$ is a left $R$-module, then $\operatorname{Pd}_{R}(A)$ $=W d_{R}(A)$. For completeness, we reproduce Eilenberg's argument in [8].

Let

$$
\rightarrow P_{n} \stackrel{d_{n}}{\rightarrow} P_{n-1} \stackrel{d_{n-1}}{\rightarrow} \cdots \rightarrow P_{0} \rightarrow A \rightarrow(0)
$$

be a projective resolution of $A$ where each $d_{n}$ is a minimal epimorphism onto the kernel of $d_{n-1}$. Then, since $d_{n}\left(P_{n}\right) \subset N P_{n-1}$ for every $n$, the sequence

$$
\rightarrow R / N \otimes_{R} P_{n} \rightarrow R / N \otimes_{R} P_{n-1} \rightarrow \cdots \rightarrow R / N \otimes_{R} P_{0} \rightarrow(0)
$$

has zero differentiation. But the homology of this complex is just $\operatorname{Tor}^{R}(R / N, A)$. Therefore, $\operatorname{Tor}_{n}^{R}(R / N, A) \cong P_{n} / N P_{n}$. Hence, if $W d_{R}(A)<n$, $\operatorname{Tor}_{n}^{R}(R / N, A)=(0)$ so $P_{n}=(0)$ and $P d_{R}<n$.

Remark. Conditions (1) and (6) of Theorem $\mathrm{P}$ are purely algebraic, yet we know of no nonhomological proof that (1) implies (6). In this connection S. Chase has found an algebraic proof that any semi-primary ring satisfies the descending chain condition on finitely generated ideals. We do not know whether perfect rings enjoy this stronger property.

3. Examples. (1) Rings with minimum condition, and, more generally, all semi-primary rings are both left and right perfect. If $R$ is left or right perfect, then $R$ is left Noetherian if and only if $R$ has left minimum condition.

(2) If $R / N$ has minimum condition and $N$ is a direct sum of two sided nilpotent ideals, then $R$ is left and right perfect, but need not be semiprimary (even when $R$ is commutative).

(3) If $R$ is left perfect, so also is the ring of $n \times n$ matrices over $R$.

(4) If $N$ is left $T$-nilpotent, then the set of all finitely nonzero matrices $\left(\left(a_{i j}\right)\right)_{i, j=1,2}, \ldots, a_{i j} \in N$, is left $T$-nilpotent. 
(5) Let $F$ be a field and let $F_{w}$ denote the algebra of all row finite matrices $\left(\left(a_{i j}\right)\right)_{i, j=1,2}, \ldots$ with coordinates in $F$. We denote by $N$ the set of all strictly lower triangular matrices in $F_{w}$ having only a finite number of nonzero coordinates, and let $R$ be the subalgebra of $F_{w}$ generated by $N$ together with the identity. Then $R$ has radical $N, R / N \cong F, N$ is left $T$-nilpotent and locally nilpotent, but $N$ is not right $T$-nilpotent. Thus, $R$ is left perfect but not right perfect.

(6) Let $R$ be a commutative ring. Then $R$ is semi-perfect if and only if $R$ is a direct sum of finitely many local rings, and $R$ is perfect if and only if, moreover, their maximal ideals are $T$-nilpotent.

If $R$ is left perfect, then Eilenberg has shown [8], essentially as in the proof of (2.6) that every projective left $R$-module is a direct sum of direct summands of $R$. We shall have occasion to use this fact below.

REMARKs. If $N$ is left $T$-nilpotent, then every finite subset of $N$ generates a nilpotent subring. In all the examples we know, however, $N$ is even nilpotent on every finitely generated right ideal $I$ in $N$, (i.e. some power of $N$ right annihilates $I$ ). One might ask whether in general every finitely generated right ideal in $N$ must be nilpotent.

Another problem has to do with the (transfinite) "Loewy length" of $N$, where the "Loewy series" of $N$ is constructed as in the proof that (7) implies (1) of Theorem P (\$1). Specifically, can this length be an arbitrary ordinal, or, say, must it be a countable one?

\section{Part II. Finitistic dimension}

4. Duality. If $A$ is a left (right) $R$-module, we write

$$
A^{*}=\operatorname{Hom}_{R}(A, R) \text {, }
$$

the dual of $A$, which is a right (left) $R$-module. As usual, the natural pairing $A \times A^{*} \rightarrow R$ defines annihilator relations between subsets of $A$ and $A^{*}$. If $S$ is a subset of $A\left(A^{*}\right)$ we denote its annihilator in $A^{*}(A)$ by $S^{\prime}$. We call $S^{\prime \prime}$ the closure of $S$ and we call a submodule $S$ of $A$ closed if $S=S^{\prime \prime}$. An elementary argument shows that $S^{\prime}$ is a closed submodule for any subset $S$ of $A$ (or $A^{*}$ ).

The pairing also provides us with a "natural" homomorphism

$$
\delta_{A}: A \rightarrow A^{* *} \text {. }
$$

By this we mean that the $\delta_{A}$ constitute natural transformations from the identity functors to ${ }^{* *}$. We call $A$ torsionless if $\delta_{A}$ is a monomorphism, reflexive if $\delta_{A}$ is an isomorphism.

For a more detailed discussion of this duality theory, we refer the reader to [6] or [13]. We shall list briefly below only those elementary facts that we shall need in the sequel. The omitted proofs can be supplied directly from the definitions.

(4.1) Given an exact sequence 


$$
(0) \rightarrow A \rightarrow B \rightarrow A / B \rightarrow(0)
$$

the dual sequence

$$
(0) \rightarrow(A / B)^{*} \rightarrow B^{*} \rightarrow A^{*}
$$

is exact, and $(A / B)^{*}$ is thus identified with $A^{\prime}$ in $B^{*}$.

(4.2) A module is torsionless if and only if every nonzero element in it can be separated from zero by a homomorphism into $R$. Therefore, if $K \subset A$, $K$ is closed in $A$ if and only if $A / K$ is torsionless.

(4.3) The dual of a finitely generated projective (free) module is a projective (free) module with the same number of generators. If $P$ is finitely generated and projective, then $P$ is reflexive, so we shall use $\delta_{P}$ to identify $P$ with $P^{* *}$.

(4.4) $K$ is a closed submodule of a free left $R$-module of rank $n(<\infty)$ if and only if $K=B^{*}$, where $B$ is a right $R$-module generated by $n$ elements.

Proof. Suppose $K$ is a closed submodule of $F$. Then, by (4.1), $K=K^{\prime \prime}$ $=\left(F^{*} / K^{\prime}\right)^{*}$, so $B=F^{*} / K^{\prime}$ works.

Conversely, given $B$, resolve, $(0) \rightarrow H \rightarrow G \rightarrow B \rightarrow(0)$ with $G$ free of rank $n$. Then, by (4.1), $B^{*}=H^{\prime}$, a closed submodule of $G^{*}$, and $G^{*}$ is free of rank $n$, by (4.3).

(4.5) A submodule of a projective module is torsionless. Conversely, if $R$ is right Noetherian, then a finitely generated torsionless left $R$-module $B$ can be embedded in a finitely generated free module.

Proof. The first statement is trivialized by (4.2). For the converse, resolve, $F \rightarrow B \rightarrow(0)$ with $F$ free of finite rank. Then $(0) \rightarrow B^{*} \rightarrow F^{*}$ embeds $B^{*}$ in a finitely generated right $R$-module, so $B^{*}$ is finitely generated ( $R$ being right Noetherian). Now resolve $B^{*}, G \rightarrow B^{*} \rightarrow(0)$, with $G$ free of finite rank. Then $(0) \rightarrow B^{* *} \rightarrow G^{*}$ embeds $B^{* *}$ in a free module of finite rank. But, since $B$ is torsionless, $\delta_{B}$ embeds $B$ in $B^{* *}$.

(4.6) In the pairing of $R$ with $R^{*}$ (=R, with operators on the opposite side), the duality annihilator relations are just the ordinary annihilator relations between left and right ideals.

(4.7) If $R$ is an integral domain, then a finitely generated $R$-module is torsionless if and only if it is torsion free. If $A$ is finitely generated and torsion free, then closed submodules are just pure submodules. If $I$ is a nonzero ideal in $R$, then $I^{*} \cong I^{-1}$.

(4.8) ("Dual Basis Lemma," [5, VII, Proposition 3.1]). A left $R$-module $P$ is projective if and only if there exist subsets $\left\{a_{i}\right\}$ of $P$ and $\left\{f_{i}\right\}$ of $P^{*}$ such that, for each $a \in P, f_{i}(a)=0$ for almost all $i$, and $a=\sum_{i} f_{i}(a) a_{i}$. Moreover, if $P$ is projective, $\left\{a_{i}\right\}$ may be taken to be any generating set of $P$.

The next result tells us when an element generates a direct summand of a free module.

(4.9) ("Unimodular Row Lemma"). Let $F$ be a free left $R$-module with 
basis $u_{1}, \cdots, u_{n}$, and let $\alpha=a_{1} u_{1}+\cdots+a_{n} u_{n} \in F$. Denote by $A$ the right ideal generated by $a_{1}, \cdots, a_{n}$. Then the following are equivalent.

(i) $A=e R$ for an idempotent $e \in R$.

(ii) $R \alpha$ is a direct summand of $F$ isomorphic to $R e$ with $\alpha \leftrightarrow e$.

Proof. (ii) is equivalent to the existence of a homomorphism $f: F \rightarrow R e$ with $f(\alpha)=e$ and $e \alpha=\alpha$. The last condition is equivalent to $A \subset e R$, and, since any homomorphism $f: F \rightarrow R$ is defined by the images $r_{i}$ of $u_{i}$, we have $e=f(\alpha)=\sum_{i} a_{i} r_{i} \in A$ from the first condition.

5. Applications to homological dimension. We define the left finitistic dimensions of $R$ as follows:

$1 G D(R)=\sup \left\{P d_{R}(A) \mid A\right.$ is a left $R$-module $\}$.

$1 F P D(R)=\sup \left\{P d_{R}(A) \mid A\right.$ is a left $R$-module with $\left.P d_{R}(A)<\infty\right\}$.

$1 F W D(R)=\sup \left\{W d_{R}(A) \mid A\right.$ is a left $R$-module with $\left.W d_{R}(A)<\infty\right\}$.

$1 F I D(R)=\sup \left\{I d_{R}(A) \mid A\right.$ is a left $R$-module with $\left.I d_{R}(A)<\infty\right\}$.

$1 f P D(R)=\sup \left\{P d_{R}(A) \mid A\right.$ is a finitely generated left $R$-module with $\left.P d_{R}(A)<\infty\right\}$.

We shall have need of

Proposition 5.1 (Auslander, [1]). $1 G D(R)=\sup \left\{P d_{R}(A) \mid A\right.$ is a finitely generated left $R$-module $\}$.

The idea of the applications to follow is that, with sufficient chain conditions, the duality theory provides an intrinsic description of the kernels that arise in the early stages of finitely generated projective resolutions. If a module has finite projective dimension, we can, by "moving out" in a resolution, reach kernels of all lower dimensions. Moreover, with appropriate chain conditions, we can guarantee that we remain in the category of finitely generated modules, if we start there.

Thus, let $A$ be a finitely generated left $R$-module, and let $(0) \rightarrow K \rightarrow P$ $\rightarrow A \rightarrow(0)$ be a resolution with $P$ finitely generated and projective. Then, by (5.1), $1 G D(R) \leqq 2$ if and only if the $K$ that arise in this way all have $P d \leqq 1$. Moreover, if $R$ is left Noetherian, we see by the above remarks that $1 f P D(R)$ $\leqq 1$ if and only if these $K$ never have $P d=1$. What are the possible modules, $K$ ? If $R$ is left Noetherian, they are all finitely generated. Hence, if $R$ is also right Noetherian, they are, by (4.5) precisely the finitely generated torsionless left $R$-modules.

Suppose now that $R$ is left and right Noetherian and we resolve, $(0) \rightarrow H$ $\rightarrow G \rightarrow K \rightarrow(0)$, with $G$ free of finite rank. Then by (4.2) and (4.4), the $H$ that arise in this way are precisely the duals of finitely generated right $R$-modules. We have now proved:

Proposition 5.2. If $R$ is left and right Noetherian, the following are equivalent.

(1) $1 G D(R) \leqq 2$.

(2) The dual of any finitely generated right $R$-module is projective. 
Proposition 5.3. If $R$ is left and right Noetherian, the following are equivalent.

(1) $1 f P D(R) \leqq 1$.

(2) If $A$ is a finitely generated right $R$-module, $A^{*}$ is projective only if $A$ is.

(3) If $A$ is a right ideal then $A^{*}$ is projective only if $A$ is.

Proof. The discussion above shows that $1 f P D(R) \leqq 1$ if and only if no finitely generated torsionless left $R$-module has $P d=1$.

(1) implies (2). Let $A$ be a finitely generated torsionless right $R$-module such that $A^{*}$ is projective, and let $(0) \rightarrow K \rightarrow F \rightarrow A \rightarrow(0)$ be a resolution with $F$ free of finite rank. Then we have $(0) \rightarrow A^{*} \rightarrow F^{*} \rightarrow F^{*} / A^{*} \rightarrow(0)$ exact with $F^{*} / A^{*}=F^{*} / K^{\prime}$ finitely generated and torsionless. Therefore, since $A^{*}$ is projective, our hypothesis forces $A^{*}=K^{\prime}$ to be a direct summand of $F^{*}$. Therefore, $K=K^{\prime \prime}$ is a direct summand of $F$, so $A$ is projective.

(2) implies (1). Let $B$ be a finitely generated torsionless left $R$-module, and suppose ( 0$) \rightarrow K \rightarrow F \rightarrow B \rightarrow(0)$ is a resolution with $F$ free of finite rank and $K$ projective. Then we must show that $K$ is a direct summand of $F$. In the dual sequence $(0) \rightarrow B^{*} \rightarrow F^{*} \rightarrow F^{*} / B^{*} \rightarrow(0), F^{*} / B^{*}=F^{*} / K^{\prime}$ is a finitely generated torsionless right $R$-module, and $\left(F^{*} / B^{*}\right)^{*}=\left(F^{*} / K^{\prime}\right)^{*}=K^{\prime \prime}=K$, by (4.1) and the fact that $B$ is torsionless. Therefore, since $K$ is projective, our hypothesis guarantees that $F^{*} / B^{*}$ is projective, so $B^{*}=K^{\prime}$ is a direct summand of $F^{*}$, and, finally, $K=K^{\prime \prime}$ is a direct summand of $F$.

(2) implies (3) is trivial, by (4.5) and the assumption that $R$ is right Noetherian.

(3) implies (2). Suppose $A$ is a finitely generated torsionless right $R$ module and $A^{*}$ is projective. Then $A^{*} \oplus B$ is free of finite rank for some finitely generated $B$. Moreover, $\left(A \oplus B^{*}\right)^{*}=A^{*} \oplus B^{* *}=A^{*} \oplus B$, so, since $A$ is projective if and only if $A \oplus B^{*}$ is, we may reduce to the case where $A^{*}$ is free of finite rank.

Now write $A^{*}=R \oplus S$ with $S$ free of one smaller rank. Then $\left(A / R^{\prime}\right)^{*}$ $=R^{\prime \prime}=R$, and $A / R^{\prime}$ is torsionless. Therefore, $\delta_{A / R^{\prime}}: A / R^{\prime} \rightarrow\left(A / R^{\prime}\right)^{* *}=R^{*}$ is a monomorphism, so $A / R^{\prime}$ is isomorphic to a left ideal. Now (3) implies $A / R^{\prime}$ is projective. Hence, $A / R^{\prime}=\left(A / R^{\prime}\right)^{* *}=R^{*}$, and $A=R^{\prime} \oplus R^{*}$. We finish by applying induction to $R^{\prime}$, since $\left(R^{\prime}\right)^{*}=S$.

REMARK. The restriction of $A$ to ideals in (5.1) (2), as was done in (5.2), is not possible since any unique factorization domain satisfies the weaker hypothesis.

By virtue of (4.7), the conditions of (5.2) become somewhat more concrete when $R$ is an integral domain.

Corollary 5.3. If $R$ is a Noetherian integral domain, the following are equivalent.

(1) $f P D(R) \leqq 1$.

(2) For any nonzero ideal $I, I^{-1}$ is invertible only when $I$ is. 
This corollary is due originally to Kaplansky, as is the following theorem, for commutative $R$. This result describes a consequence of the condition $1 f P D(R)=0$.

THEOREM 5.4. The following are equivalent for any ring $R$.

(1) A finitely generated projective submodule of a projective left $R$-module is always a direct summand.

(2) The left annihilator of a finitely generated proper right ideal is always nonzero.

(3) $(P / K)^{*} \neq(0)$ whenever $P$ is a finitely generated projective right $R$-module and $K$ is a finitely generated proper submodule.

Proof. (1) implies (2). Let $A=\left(a_{1}, \cdots, a_{n}\right)$ be a finitely generated right ideal with no left annihilator. If $F$ is a free left $R$-module with basis $u_{1}, \cdots, u_{n}$, and $\alpha=a_{1} u_{1}+\cdots+a_{n} u_{n} \in F$, then $R \alpha \cong R$ (with $\alpha \leftrightarrow 1$ ). By (1), therefore, $R \alpha$ is a direct summand of $F$, so, by the "unimodular row lemma" (4.9), $A=R$.

(2) implies (3). By adding a finitely generated projective module to $P$ and $K$, if necessary, we may assume $P$ is free. After successive removal of basis elements lying in $K$ we may assume that, relative to some basis of $P$, $K$ contains no element with first coordinate 1 . Thus, we may write $P=R \oplus S$, where, if $\pi$ is the projective of $P$ onto $R, \pi(K) \neq R$. Therefore, since $\pi(K)$ is finitely generated, (2) provides us with an $r \neq 0$ such that $r \pi(K)=(0)$. Now define $f: P \rightarrow R$ by $f(x)=r \pi(x)$. Then $f$ induces a nonzero element of $(P / K)^{*}$.

We shall need a lemma before concluding the proof.

LEMMA 5.5. Let $K$ be a finitely generated submodule of a finitely generated projective right $R$-module $S$. Then (5.4) (3) implies that $K^{\prime}$ is a direct summand of $S^{*}$ only when $K$ is a direct summand of $S$.

Proof. If $K^{\prime}$ is a direct summand of $S^{*}$, then $K^{\prime \prime}$ is a direct summand of $S$. Therefore, $K^{\prime \prime}$ is finitely generated and projective. Moreover, since any homomorphism $K^{\prime \prime} \rightarrow R$ can be extended to $S$, we see that $\left(K^{\prime \prime} / K\right)^{*}=(0)$. Therefore, by (5.4) (3), $K=K^{\prime \prime}$, a direct summand of $S$.

(3) implies (1). Let $(0) \rightarrow Q \rightarrow P$ be exact with $P$ and $Q$ projective left $R$ modules and $Q$ finitely generated. By adding a complement to make $P$ free and then reducing to a finitely generated direct summand containing $Q$, we may assume also that $P$ is finitely generated. We have the "restriction" map $P^{*} \rightarrow Q^{*}$, and since every nonzero element of $Q$ is separated from zero by a homomorphism from $P$ to $R$, (im $\left.P^{*}\right)^{\prime}=(0)$, a direct summand of $Q^{* *}=Q$. Therefore, since im $P^{*}$ is finitely generated, (5.5) allows us to conclude that $\operatorname{im} P^{*}$ is a direct summand of $Q^{*}$. Hence, im $P^{*}=\left(\operatorname{im} P^{*}\right)^{\prime \prime}=(0)^{\prime}=Q^{*}$; i.e. every homomorphism from $Q$ to $R$ can be extended to $P$.

Now take $a_{1}, \cdots, a_{n} \in Q$ and $f_{1}, \cdots, f_{n} \in Q^{*}$ as in the Dual Basis Lemma (4.8), and let $g_{i}$ be an extension of $f_{i}$ to $P$. We define $g: P \rightarrow P$ by $g(a)$ 
$=\sum_{i} g_{i}(a) a_{i}$. Then $g$ is idempotent with range $Q$, so $Q$ is a direct summand of $P$.

Corollary 5.6. Consider the conditions:

(1) $1 f P D(R)=0$.

(2) A proper finitely generated right ideal in $R$ has nonzero left annihilator. Then (1) always implies (2), and if $R$ is left Noetherian they are equivalent.

6. The condition $1 F P D(R)=0$.

Lemma 6.1. Suppose that $R$ is left perfect and that $R$ satisfies the conditions of Theorem 5.4. Then $1 F P D(R)=0$.

Proof. We must show that, if $Q \subset P$ with $P$ and $Q$ projective left $R$-modules, then $Q$ is a direct summand of $P$. Since $R$ is left perfect, $Q$ is a direct sum of cyclic modules (see $\S 3$ ), and, by our hypothesis, every finitely generated direct summand of $Q$ is a direct summand of $P$. Therefore, $Q$ is a direct limit of direct summands of $P$, so $P / Q$ is a direct limit of projective modules. Therefore, since $R$ is left perfect, $P / Q$ is projective, by (5) of Theorem $P$.

The following result was proved by $\mathrm{S}$. Chase by dualizing an argument used in proving (7.1) of the following section.

LEMma 6.2. If $R$ is left perfect, then $1 F P D(R)=0$ if and only if every simple left $R$-module is a homomorphic image of an injective module.

Proof. Suppose $1 F P D(R)=0$ and let $S$ be a simple left $R$-module. Then we have the following commutative diagram,

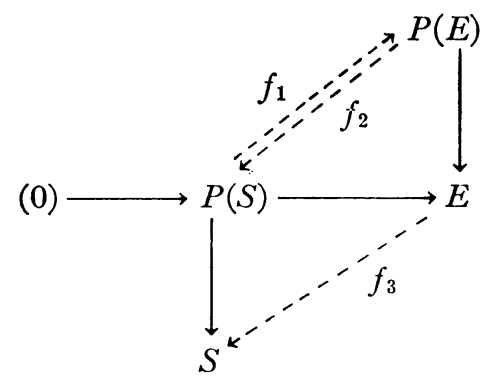

with $E$ injective, where $f_{1}$ exists because $P(S)$ is projective, $f_{2}$ exists because $f_{1}$ is a monomorphism and $1 F P D(R)=0$, and $f_{3}$ exists since

$$
\operatorname{ker}(P(E) \rightarrow E) \subset N P(E) \subset \operatorname{ker}(P(E) \rightarrow S),
$$

where $N$ is the $J$-radical of $R$, the last inclusion following from the simplicity of $S$.

Conversely, suppose given

$$
(0) \rightarrow K \rightarrow P(B) \rightarrow B \rightarrow(0)
$$


with $K$ projective. We must show $K=(0)$. If not, there exists an epimorphism $K \rightarrow S \rightarrow(0)$ with $S$ simple (since $K \neq N K$ and $K / N K$ is completely reducible), and, by hypothesis, an epimorphism $E \rightarrow S \rightarrow(0)$ with $E$ injective. Now we have the commutative diagram

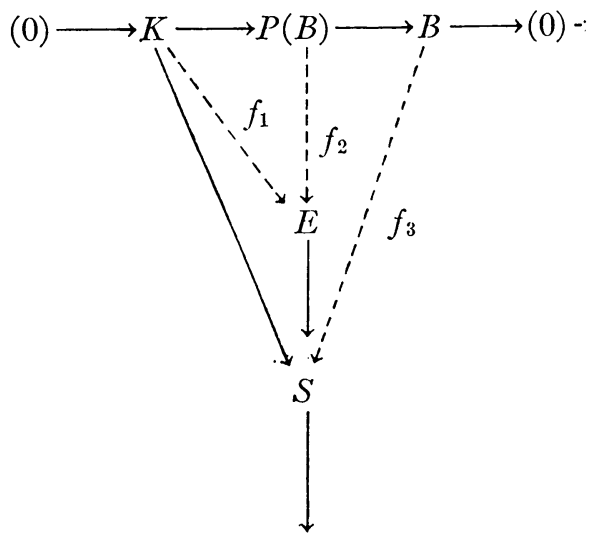

(0)

where $f_{1}$ exists since $K$ is projective, $f_{2}$ because $E$ is injective, and $f_{3}$ exists because

$$
\operatorname{ker}(P(B) \rightarrow B) \subset N P(B) \subset \operatorname{ker}(P(B) \rightarrow S) .
$$

But then $K \rightarrow S$ can be factored through $K \rightarrow P(B) \rightarrow B$, the zero map; contradiction.

THEOREM 6.3. The following are equivalent for any ring $R$.

(1) $1 F P D(R)=0$.

(2) $R$ is left perfect and $1 f P D(R)=0$.

(3) $R$ is left perfect and every finitely generated proper right ideal has nonzero left annihilator.

(4) $R$ is left perfect and every simple left $R$-module is a homomorphic image of an injective module.

Proof. Suppose that $R$ is left perfect. Then (1) implies (2) trivially, (2) implies (3) by (5.6), and (3) implies (1) by (6.1). Moreover, (1) $\Leftrightarrow(4)$ by (6.2). Therefore, it remains only for us to show that if $1 F P D(R)=0$, then $R$ is left perfect.

Let $\left\{a_{1} \cdots a_{n} R\right\}$ be a decreasing chain of principal right ideals in $R$. Form $\left[F,\left\{a_{n}\right\}, G\right]$ as in $\S 1$. Since $P d_{R}(F / G) \leqq 1$, by (1.1), our hypothesis guarantees that $P d_{R}(F / G)=0$, so $G$ is a direct summand of $F$. Therefore, by (1.3), the chain terminates.

7. The condition $1 F I D(R)=0$. Throughout this section all modules will be left $R$-modules, all ideals left ideals, and all sequences exact. Our object is to prove the following result, together with several interesting corollaries. 
THEOREM 7.1. For any ring $R$ the following conditions are equivalent.

(a) $1 F I D(R)=0$.

(b) Every injective left $R$-module is the injective envelope of a submodule of a projective module.

(c) Every nonzero left $R$-module contains a nonzero submodule isomorphic to a (principal) ideal.

(d) Every injective left $R$-module is isomorphic to the injective envelope of a direct sum of principal ideals.

To prove this theorem we exploit heavily the theorem of EckmannSchopf (see Introduction), so we first review the fundamental properties of injective envelopes. If $A$ is a submodule of $B$ we denote by $A \subset_{e} B$ the fact that $A$ is essential in $B$ (see $\S 2$ ). Recall, an injective envelope $E(A)$ of $A$ is defined by the conditions: $A \subset_{e} E(A)$ and $E(A)$ is injective.

The following lemma is essentially the dual of (2.3).

(7.2) If $A \subset F$ and $F$ is injective, then we can write $F=E(A) \oplus F^{\prime}$.

Proof. There exists a homomorphism $E(A) \rightarrow F$ extending the identity on $A$, because $F$ is injective; since $A \subset_{e} E(A)$ this must be a monomorphism. Therefore, since $E(A)$ is injective, its image must be a direct summand of $F$.

(7.3) If $A \subset C$ and $B \subset_{e} C$, then $(A \cap B) \subset_{e} A$.

Proof. Suppose $0 \neq \alpha \in A$. Since $B C_{e} C$ there exists an $r \in R$ such that $0 \neq r \alpha \in B$. But then $r \alpha \in A \cap B$.

(7.4) If $C \subset_{e} B$ and $A \rightarrow{ }^{\pi} B \rightarrow(0)$, then $\pi^{-1}(C) \subset_{e} A$.

Proof. Suppose $\alpha \in A, \alpha \notin \pi^{-1}(C)$. Then $\pi(\alpha) \neq 0$ so there exists an $r \in R$ such that $0 \neq r \pi(\alpha)=\pi(r \alpha) \in C$. Therefore, $0 \neq r \alpha \in \pi^{-1}(C)$.

Now we can initiate the proof of Theorem 7.1.

(a) implies (b). Let $E$ be any injective module and let $P \rightarrow E \rightarrow(0)$ be a resolution with $P$ projective. Then we have a commutative diagram

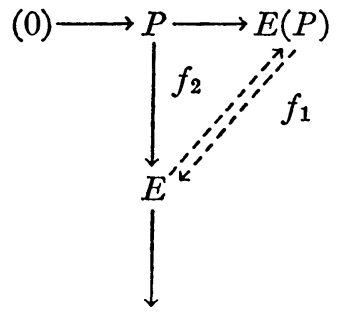

$(0)$

where $f_{1}$ exists since $E$ is injective, and $f_{2}$ exists because $f_{1}$ is an epimorphism and $1 F I D(R)=0$. Thus, identifying $E$ with a direct summand of $E(P)$, we can write $E(P)=E \oplus F$. Then, since $P \subset_{e} E(P),(7.3)$ tells us that $(P \cap E) \subset_{e} E$, so $E=E(P \cap E)$.

To prove $(\mathrm{b}) \Rightarrow(\mathrm{a})$ we need 
Lemma 7.5. If $F \rightarrow^{\pi} E(P) \rightarrow(0)$ with $F$ injective and $P$ projective, then $\pi$ splits.

Remark. This lemma can be thought of as describing the projective objects in the category of injective modules.

Proof. Let $Q=\pi^{-1}(P) . \pi \mid Q: Q \rightarrow P \rightarrow(0)$ so, since $P$ is projective, $Q=K \oplus P^{\prime}$ where $K=\operatorname{ker}(\pi \mid Q)=\operatorname{ker}(\pi)$ and $\pi$ induces an isomorphism of $P^{\prime}$ onto $P$. By (7.4) $Q \subset_{e} F$; therefore, $F=E(Q)=E(K) \oplus E\left(P^{\prime}\right)$. Since $\pi \mid P^{\prime}$ is an isomorphism, the uniqueness property of the injective envelope guarantees that $\pi$ maps $E\left(P^{\prime}\right)$ isomorphically onto $E(P)$. Now let $\alpha \in E(K)$; we may choose $\beta \in E\left(P^{\prime}\right)$ such that $\pi(\alpha)=\pi(\beta)$. Therefore $\alpha-\beta \in K \subset E(K)$ so $\beta \in E(K) \cap E\left(P^{\prime}\right)=(0)$. Therefore $\pi(\alpha)=\pi(0)=0$, so $\alpha \in K$; i.e. $K=E(K)$, a direct summand of $F$.

(b) implies (a). Suppose $F \rightarrow^{\pi} E \rightarrow(0)$ with $F$ and $E$ injective; we must show that $\pi$ splits. There exists, by (b), a projective module $P$ and a submodule $S \subset P$ such that $E=E(S)$. By (7.2), $E(P)=E(S) \oplus E^{\prime}$ for some $E^{\prime}$. Hence, by adding $E^{\prime}$ to both $F$ and $E$ and making $\pi$ the identity on $E^{\prime}$, we may assume $E$ has the form $E(P)$ with $P$ projective. Now we are in the setting of (7.5).

We have thus shown $(a) \Leftrightarrow(b)$. We shall now prepare to prove $(b) \Rightarrow(c)$ $\Leftrightarrow(d) \Rightarrow(b)$. To facilitate the arguments we introduce an auxiliary concept.

Definition. Let $\Sigma$ be a nonvacuous family of nonzero cyclic $R$-modules. A $\Sigma$-module is a nonzero direct sum of modules each of which is isomorphic to a member of $\Sigma$. $\Sigma$ is called an injective basis $(I B)$ for $R$ if every nonzero injective $R$-module contains a $\Sigma$-module.

ExAmple (7.6) $\Sigma=\{R / I \mid I$ is any proper left ideal $\}$ is an $I B$.

Example (7.7) If $R$ is right perfect then $\Sigma=\{R / M \mid M$ is any maximal left ideal $\}$ is an $I B$.

Proof. This follows from condition (7) of Theorem P.

Example (7.8) (Matlis, [12]). If $R$ is commutative and Noetherian, then $\Sigma=\{R / P \mid P$ is any prime ideal $\}$ is an $I B$.

Proof. Let $A$ be any nonzero $R$-module. Choose $\alpha \neq 0$ in $A$ such that $((0): \alpha)=P$ is maximal. Then $P$ must be a prime. For suppose $r s \in P$ and $s \notin P$. Then $r \in((0): s \alpha) \subset P$, but, by the maximality of $P$ (note that $s \alpha \neq 0$ ) we have $((0): s \alpha)=P$.

The existence of bases for vector spaces is generalized in this context by

Proposition 7.9. If $\Sigma$ is an IB for $R$, then every nonzero injective $R$-module is the injective envelope of a $\Sigma$-module. In particular, for any ring $R$, every injective R-module is the injective envelope of a direct sum of cyclic modules.

Proof. Let $E$ be a nonzero injective module. Consider subsets $X$ of $E$ satisfying (i) $R x$ is a $\Sigma$-module for each $x$ in $X$, and (ii) the submodule generated by $X$ is $\Sigma_{x} \oplus R x$. These sets are inductively ordered by inclusion, so we may take a maximal such set $X$. If $D$ is the $(\Sigma-)$ module generated by $X$ 
then, by (7.2), we may write $E=E(D) \oplus F$ for some injective module $F$. If $F \neq(0)$ then $F$ contains a $\Sigma$-module, by hypothesis. In particular, there is an element $y \in F$ such that $R y$ is a $\Sigma$-module. Then $X \cup\{y\}$ contradicts the maximality of $X$. Therefore $F=(0)$ and $E=E(D)$.

REMARK. In view of (7.9), condition (4) of Theorem (7.1) amounts to saying that the principal ideals in $R$ constitute an $I B$.

Lemma 7.10. A nonzero submodule of a projective module contains a nonzero submodule isomorphic to a principal ideal.

Proof. The lemma easily reduces to showing that if $F$ is a free module of finite rank and $0 \neq \alpha \in F$, then $R \alpha$ contains a nonzero submodule isomorphic to an ideal. Let us write the elements of $F$ as $n$-tuples; then $\alpha=\left(a_{1}, \cdots, a_{n}\right)$. We proceed by induction on the number of nonzero coordinates of $\alpha$. If $\alpha=\left(a_{1}, 0, \cdots, 0\right)$ then $R \alpha \cong R a_{1}$. After a permutation of the basis, now, we may assume $a_{1}, \cdots, a_{k} \neq 0, a_{k+1}=\cdots=a_{n}=0$. Then if $\left((0): a_{1}\right)=\cdots$ $=\left((0): a_{k}\right), \quad R \alpha \cong R a_{1}$. Therefore, say $\left((0): a_{k}\right) \nsubseteq\left((0): a_{1}\right)$. Then choosing $r \in\left((0): a_{k}\right), r \notin\left((0): a_{1}\right)$ we see that $0 \neq r \alpha$ and $r \alpha$ has fewer nonzero coordinates, so we finish by induction in $\operatorname{Rr} \alpha$.

We now easily conclude the proof of Theorem 7.1.

(b) implies (c). Let $A$ be a nonzero $R$-module. Then $E(A)=E(S)$ where $S \subset P$ for some projective module $P$. By (7.3), $(A \cap S) \subset{ }_{e} A$, so $A \cap S \neq(0)$. Therefore, by (7.10), $A \cap S$ contains a nonzero submodule isomorphic to an ideal.

(c) implies (d). Condition (c) clearly implies that the set of nonzero principal ideals is an $I B$. Proposition (7.9) then proves (d).

(d) implies (b) is trivial since a direct sum of ideals is clearly embeddable in a projective module. QED.

Now suppbse $1 F I D(R)=0$ and that $A$ is a nonzero $R$-module such that every nonzero cyclic submodule of $A$ is isomorphic to $A$. Then (c) implies that $A$ is embeddable in $R$. We have thus proved

Corollary 7.11. If $1 F I D(R)=0$, then every simple left $R$-module is isomorphic to an ideal; i.e. every left ideal has nonzero right annihilator.

Corollary 7.12. If $R$ is commutative and $F I D(R)=0$, then $R / P$ is isomorphic to an ideal for every prime $P$.

These corollaries can be reversed in special cases.

COROLlaRY 7.13. If $R$ is right perfect the following are equivalent.

(1) $1 F I D(R)=0$.

(2) Every simple left $R$-module is isomorphic to an ideal.

(3) Every left ideal has nonzero right annihilator.

Proof. The equivalence of (2) and (3) is trivial and (1) implies (2) by (7.11). But (2) together with example (7.7) clearly implies that the principal 
ideals in $R$ constitute an $I B$.

Corollary 7.14. If $R$ is commutative and Notherian, then $F I D(R)=0$ if and only if every prime in $R$ belongs to (0). Thus, FID $(R)=0$ implies that $R$ has only finitely many primes, so the Krull dimension of $R$ is at most one.

Proof. Example (7.8) shows that if $R / P$ is embeddable in $R$ for every prime $P$ then the principal ideals in $R$ constitute an $I B$. The last statements are well known from Noetherian ideal theory.

Remark. Corollary (7.14) suggests, for $R$ commutative and Noetherian, that $F I D(R) \geqq($ Krull dimension of $R)-1$. This fact is proved in [4].

8. Comparison of finitistic dimensions. Examples. Let $R$ be a ring and let $K$ denote the rational numbers modulo 1 . If $B$ is an abelian group we write $B^{*}=\operatorname{Hom}_{Z}(B, K)$; when $B$ is a left $R$-module $B^{*}$ is a right $R$-module. The duality isomorphism [5, VI, Proposition 5.1] then asserts, in the situation $\left(A_{R},{ }_{R} B\right)$, that

$$
\operatorname{Ext}_{R}\left(A, B^{*}\right)=\operatorname{Tor}^{R}(A, B)^{*} .
$$

From this we conclude that $W d_{R}(B)=I d_{R}\left(B^{*}\right)$, and hence $1 F W D(R)$ $\leqq r F I D(R)$.

Now suppose that $R$ is left Noetherian and we are in the situation $\left(A_{R}, B_{R}\right)$ with $A$ finitely generated. Then the duality isomorphism [5, VI, Proposition 5.3] asserts that

$$
\operatorname{Tor}^{R}\left(B^{*}, A\right)=\operatorname{Ext}_{R}(A, B)^{*} .
$$

But it is sufficient, when computing injective dimension, to test $B$ against finitely generated modules. We therefore conclude that $I_{R}(B)=W d_{R}\left(B^{*}\right)$, and consequently $1 F W D(R) \geqq r F I D(R)\left({ }^{3}\right)$.

These results suggest comparing $1 F P D(R)$ and $1 F W D(R)$ with $r F I D(R)$ rather than $1 F I D(R)$. In fact, examples of S. Chase and of Rosenberg and Zelinsky show that, even for finite dimensional algebras, $1 F P D(R)$ $(=1 F W D(R))$ and $1 F I D(R)$ can differ more or less arbitrarily. However, the conclusions above, together with some elementary considerations, allow us to determine the following inequalities.

(8.1) General $R$

$$
\begin{aligned}
1 G D(R) & \geqq 1 F P D(R) \geqq 1 f P D(R), \\
r F I D(R) & \geqq 1 F W D(R) .
\end{aligned}
$$

(8.2) $R$ left Noetherian

$$
1 G D(R) \geqq\left\{\begin{array}{c}
1 F P D(R) \\
r F I D(R)=1 F W D(R)
\end{array}\right\} \geqq 1 f P D(R) .
$$

(3) These applications of the duality homomorphisms are due to E. Matlis (oral communication). 
(8.3) $R$ left perfect

$$
1 G D(R) \geqq r F I D(R) \geqq 1 F P D(R)=1 F W D(R) \geqq 1 f P D(R) .
$$

(8.4) $R$ left Artinian (=left minimum condition)

$$
1 G D(R) \geqq r F I D(R)=1 F P D(R)=1 F W D(R) \geqq 1 f P D(R) .
$$

A number of questions regarding the completeness of these inequalities can be raised. For example, let $R$ be left perfect. Then one might inquire whether the chain condition is necessary for the equality of $f F I D(R)$ and $1 F P D(R)$. Our results permit us to answer this affirmatively. For, by (6.3), $1 F P D(R)=0$ if and only if every finitely generated proper right ideal has nonzero left annihilator, and, by (7.13), $r F I D(R)=0$ if and only if every proper right ideal has nonzero left annihilator.

Example 8.5 (Chase). The ring $R$ below has the following properties:

(i) $R$ is semi-primary (the cube of the radical is zero).

(ii) $R$ has an involution.

(iii) Every finitely generated proper right ideal in $R$ has nonzero left annihilator and

(iv) $R$ has a maximal right ideal with zero left annihilator. Hence

$$
r F I D(R)>1 F P D(R)=0, \text { and } 1 F I D(R)>r F P D(R)=0 \text {. }
$$

Let $K$ be a field, $V$ an infinite dimensional $K$-space with a nondegenerate symmetric inner product [ , ]: $V \times V \rightarrow K$, and let $S$ be the truncated polynomial ring $K[x] /\left(x^{2}\right)$. Then $R$ is the ring of all $2 \times 2$ "matrices"

$$
\left(\begin{array}{cc}
a & v \\
u & f(x)
\end{array}\right), \quad a \in K, u, v \in V, f(x) \in S,
$$

with coordinate wise addition and multiplication defined by

$$
\left(\begin{array}{cc}
a & v \\
u & f(x)
\end{array}\right)\left(\begin{array}{cc}
a^{\prime} & v^{\prime} \\
u^{\prime} & f^{\prime}(x)
\end{array}\right)=\left(\begin{array}{cc}
a a^{\prime} & a v^{\prime}+v f^{\prime}(0) \\
u a^{\prime}+f(0) u^{\prime} & f(x) f^{\prime}(x)+[u, v] x
\end{array}\right) .
$$

The associativity depends on the fact that, for $f(x) \in S, x f(x)=x f(0)$.

Rosenberg and Zelinsky have raised the following questions, which are unanswered even for finite dimensional algebras: (i) Is $1 F P D(R)=1 f P D(R)$ ? And (ii) is $1 f P D(R)$ finite? Question (i) is answered affirmatively, even for left perfect rings, when $1 f P D(R)=0$, by (6.3).

If $R$ is commutative, then our results show easily that $F P D(R)=0$ implies $F I D(R)=0$. It seems plausible that, for $R$ commutative, one always has $F P D(R) \geqq F I D(R)$. This is indeed true if $R$ is also Noetherian, and is proved in [4]. Moreover, the example below shows that the inequality may be strict, so this, together with example (8.5) shows that there is no universal inequality relating $1 F P D(R)$ to $r F I D(R)$. 
ExAmple 8.6. The ring $R$ below has the following properties:

(i) $R$ is commutative with an infinite set of orthogonal idempotents.

(ii) $F P D(R)>F I D(R)=0$.

Let $S$ be the ring of all sequences $\left(a_{0}, a_{1}, \cdots, a_{n}, \cdots\right)$ with coordinates in the integers modulo four (with pointwise operations). If $e_{n}$ is the sequence with 1 in the $n$th place and zeroes elsewhere, we take $R$ to be the subring of $S$ generated by $\left\{e_{n} \mid n=1,2, \cdots\right\}$, the identity, and the sequence $(2,0, \cdots, 0, \cdots)$. (Note that $e_{0} \in R$.)

Finally, for examples of semi-primary rings $R$ with $1 F I D(R)=0$ (and hence also $\operatorname{rFPD}(R)=0$ ), we refer the reader to Auslander [1, Propositions 14 and $15]$. In particular, these include all quasi-Frobenius rings.

\section{BIBLIOGRAPHY}

1. M. Auslander, On the dimension of modules and algebras, III, Nagoya Math. J. vol. 9 (1955) pp. 67-77.

2. - On regular group rings, Proc. Amer. Math. Soc. vol. 8 (1957) pp. 658-664.

3. M. Auslander and D. Buchsbaum, Homological dimension in local rings, Trans. Amer. Math. Soc. vol. 85 (1957) pp. 390-405.

4. H. Bass, Finitistic dimension in Noetherian rings, (in preparation.)

5. H. Cartan and S. Eilenberg, Homological algebra, Princeton University Press, 1956. 354.

6. J. Dieudonné, Remarks on quasi-Frobenius rings, Illinois J. Math. vol. 2 (1958) pp. 346-

7. B. Eckmann and A. Schopf, Über injective moduln, Arch. Math. vol. 4 (1953) pp. 75-78.

8. S. Eilenberg, Homological dimension and syzygies, Ann. of Math. vol. 64 (1956) pp. 328336.

9. S. Eilenberg and T. Nakayama, On the dimension of modules and algebras, V, Nagoya Math. J. vol. 11 (1957) pp. 9-12.

10. M. Harada, Note on the dimension of modules and algebras, J. Inst. Polytech. Osaka City Univ. Ser. A, vol. 7 (1956) pp. 17-27. 1956.

11. N. Jacobson, Structure of rings, Amer. Math. Soc. Colloquium Publications, vol. 37,

12. E. Matlis, Injective modules over Noetherian rings, Pacific J. Math. vol. 8 (1958) pp. 511-528.

13. K. Morita, Duality for modules and its applications to the theory of rings with minimum condition, Science Reports, Tokyo Kyoiku Daigaku, vol. 6, 1958, pp. 83-142.

14. J. P. Serre, Sur la dimension homologique des anneaux et des modules Noethériens, Proceedings International Symposium on Algebraic Number Theory, Tokyo and Nikko, 1955, pp. 175-189.

University of Chicago, Chicago, Illinois 